\title{
RELAXING THE NONSINGULARITY ASSUMPTION FOR INTERVALS OF TOTALLY NONNEGATIVE MATRICES*
}

\author{
MOHAMMAD ADM ${ }^{\dagger}$, KHAWLA AL MUHTASEB ${ }^{\ddagger}$, AYED ABEDEL GHANI $^{\S}$, AND JÜRGEN GARLOFF ${ }^{\boldsymbol{q}}$
}

\begin{abstract}
Totally nonnegative matrices, i.e., matrices having all their minors nonnegative, and matrix intervals with respect to the checkerboard partial order are considered. It is proven that if the two bound matrices of such a matrix interval are totally nonnegative and satisfy certain conditions, then all matrices from this interval are totally nonnegative and satisfy these conditions, too, hereby relaxing the nonsingularity condition in the former paper [M. Adm and J. Garloff. Intervals of totally nonnegative matrices. Linear Algebra Appl., 439:3796-3806, 2013.].
\end{abstract}

Key words. Matrix interval, Checkerboard partial order, Totally nonnegative matrix, Cauchon matrix, Cauchon Algorithm, Descending rank conditions.

AMS subject classifications. 15B48.

1. Introduction. A real matrix is called totally nonnegative if all its minors are nonnegative. Such matrices arise in a variety of ways in mathematics and its applications. For background information the reader is referred to the monographs [9] and [15]. In [2], the following interval property was shown: Consider the checkerboard order which is obtained from the usual entry-wise order on the set of the square real matrices of fixed order by reversing the inequality sign for each entry in a checkerboard fashion. If the two bound matrices of an interval with respect to the checkerboard order are nonsingular and totally nonnegative, then all matrices lying between the two bound matrices are nonsingular and totally nonnegative, too. The purpose of this paper is to relax the nonsingularity assumption on the two bound matrices and to allow rectangular matrices instead of square matrices. For a collection of various classes of matrices which enjoy an interval property, see [11].

We mention a closely related problem, viz. given a totally nonnegative matrix, find for each of its entries the maximum allowable perturbation such that the perturbed matrix remains totally nonnegative. This problem was solved in [3] for the tridiagonal totally nonnegative and in [7] for the general totally nonnegative matrices. For the totally positive matrices, i.e., matrices having all their minors positive (here the perturbed matrix has in turn to be totally positive), it was established in [10], see also [9, Section 9.5], for a few specified entries and in [6] for arbitrary entries. The similar problem for a uniform perturbation of all the coefficients of a totally positive matrix was considered in [13, Section 7].

The organization of our paper is as follows. In Section 2, we introduce our notation and give some auxiliary results which we use in the subsequent sections. In Section 3, we recall the condensed form of the

\footnotetext{
${ }^{*}$ Received by the editors on July 17, 2019. Accepted for publication on December 18, 2019. Handling Editor: Michael Tsatsomeros. Corresponding Author: Jürgen Garloff.

${ }^{\dagger}$ Department of Applied Mathematics and Physics, Palestine Polytechnic University, Hebron, Palestine (mjamathe@yahoo.com, moh_95@ppu.edu).

${ }_{\ddagger}$ Department of Applied Mathematics and Physics, Palestine Polytechnic University, Hebron, Palestine (khawla@ppu.edu).

$\S$ Department of Applied Mathematics and Physics, Palestine Polytechnic University, Hebron, Palestine (ayed42@ppu.edu).

IInstitute for Applied Research, University of Applied Sciences / HTWG Konstanz, D-78405 Konstanz, Germany, and Department of Mathematics and Statistics, University of Konstanz, Konstanz, D-78464 Konstanz, Germany (juergen.garloff@htwg-konstanz.de).
} 
Cauchon Algorithm and some of its properties. In Section 4, we present our new results on the application of the Cauchon Algorithm, and apply them in the last section to the above mentioned interval problem.

\section{Notation and auxiliary results.}

2.1. Notation. We introduce the notation used in our paper. For integers $n, m, \kappa$, we denote by $\mathcal{S}$ the set $\{1, \ldots, n-1\} \times\{1, \ldots, m-1\}$, and by $Q_{\kappa, n}$ the set of all strictly increasing sequences of $\kappa$ integers chosen from $\{1,2, \ldots, n\}$. Let $A$ be a real $n$-by-m matrix. For $\alpha=\left\{\alpha_{1}, \alpha_{2}, \ldots, \alpha_{\kappa}\right\} \in Q_{\kappa, n}, \beta=\left\{\beta_{1}, \beta_{2}, \ldots, \beta_{\mu}\right\} \in$ $Q_{\mu, m}$, we denote by $A[\alpha \mid \beta]$ the $\kappa$-by- $\mu$ submatrix of $A$ contained in the rows indexed by $\alpha_{1}, \alpha_{2}, \ldots, \alpha_{\kappa}$ and columns indexed by $\beta_{1}, \beta_{2}, \ldots, \beta_{\mu}$. We suppress the curly brackets when we enumerate the indices explicitly. A measure of the gaps in an index sequence $\alpha \in Q_{\kappa, n}$ is the dispersion of $\alpha$, denoted by $d(\alpha)$, which is defined by $d(\alpha):=\alpha_{\kappa}-\alpha_{1}-\kappa+1$. If $d(\alpha)=0$, we call $\alpha$ contiguous, if $d(\alpha)=d(\beta)=0$, we call the submatrix $A[\alpha \mid \beta]$ contiguous, and in the case $\kappa=\mu$, we call the corresponding minor contiguous. For any contiguous $\kappa$-by- $\kappa$ submatrix $A[\alpha \mid \beta]$ of $A$, we call the submatrix

$$
A\left[\alpha_{1}, \ldots, \alpha_{\kappa}, \alpha_{\kappa}+1, \ldots, n \mid 1, \ldots, \beta_{1}-1, \beta_{1}, \ldots, \beta_{\kappa}\right]
$$

of $A$ having $A[\alpha \mid \beta]$ in its upper right corner the left shadow of $A[\alpha \mid \beta]$, and, analogously, we call the submatrix

$$
A\left[1, \ldots, \alpha_{1}-1, \alpha_{1}, \ldots, \alpha_{\kappa} \mid \beta_{1}, \ldots, \beta_{\kappa}, \beta_{\kappa}+1, \ldots, m\right]
$$

having $A[\alpha \mid \beta]$ in its lower left corner the right shadow of $A[\alpha \mid \beta]$. By $E_{i j}$ we denote the matrix in $\mathbb{R}^{n, m}$ which has in position $(i, j)$ a one, while all other entries are zero. A matrix $A \in \mathbb{R}^{n, m}$ is called totally nonnegative (abbreviated $T N$ henceforth) if $\operatorname{det} A[\alpha \mid \beta] \geq 0$, for all $\alpha, \beta \in Q_{\kappa, n^{\prime}}, \kappa=1,2, \ldots, n^{\prime}$, where $n^{\prime}:=\min \{n, m\}$. If a totally nonnegative matrix is also nonsingular, we write $N s T N$. If $n=m$, we set $A^{\#}:=T A T$, where $T=\left(t_{i j}\right)$ is the permutation matrix of order $n$ (antidiagonal matrix) with $t_{i j}:=\delta_{i, n-j+1}, i, j=1, \ldots, n$. If $A$ is $T N$, then $A^{\#}$ is $T N$, too, e.g., [9, Theorem 1.4 .1 (iii)].

We endow $\mathbb{R}^{n, m}$ with two partial orders: Firstly, with the usual entry-wise partial order: For $A=\left(a_{k j}\right)$, $B=\left(b_{k j}\right) \in \mathbb{R}^{n, m}$

$$
A \leq B: \Leftrightarrow a_{i j} \leq b_{i j}, i=1, \ldots, n, j=1, \ldots, m
$$

Secondly, with the checkerboard partial order, which is defined as follows

$$
A \leq{ }^{*} B: \Leftrightarrow(-1)^{i+j} a_{i j} \leq(-1)^{i+j} b_{i j}, i=1, \ldots, n, j=1, \ldots, m
$$

We denote by $\mathbb{I}\left(\mathbb{R}^{n, m}\right)$ the set of all matrix intervals of order $n$-by- $m$ with respect to the checkboard partial order

$$
[A, B]:=\left\{Z \in \mathbb{R}^{n, m} \mid A \leq^{*} Z \leq^{*} B\right\}
$$

2.2. Auxiliary results. In this subsection, we list some facts that will be employed in Sections 4 and 5. We will often make use of the following determinantal identity.

Lemma 2.1. (Sylvester's Determinantal Identity, see, e.g., [9, pp. 29-30]) Partition $A \in \mathbb{R}^{n, n}$, $n \geq 3$, as follows:

$$
A=\left(\begin{array}{ccc}
c & A_{12} & d \\
A_{21} & A_{22} & A_{23} \\
e & A_{32} & f
\end{array}\right)
$$


where $A_{22} \in \mathbb{R}^{n-2, n-2}$ and $c, d, e, f$ are scalars. Define the submatrices

$$
\begin{array}{ll}
C:=\left(\begin{array}{cc}
c & A_{12} \\
A_{21} & A_{22}
\end{array}\right), \quad D:=\left(\begin{array}{cc}
A_{12} & d \\
A_{22} & A_{23}
\end{array}\right), \\
E:=\left(\begin{array}{cc}
A_{21} & A_{22} \\
e & A_{32}
\end{array}\right), \quad F:=\left(\begin{array}{cc}
A_{22} & A_{23} \\
A_{32} & f
\end{array}\right) .
\end{array}
$$

Then, if $\operatorname{det} A_{22} \neq 0$, the following relation holds

$$
\operatorname{det} A=\frac{\operatorname{det} C \operatorname{det} F-\operatorname{det} D \operatorname{det} E}{\operatorname{det} A_{22}} .
$$

The following two lemmas provide information on the rank of certain submatrices of $T N$ matrices.

Lemma 2.2. ([9, Theorem 7.2.8]) Suppose that $A \in \mathbb{R}^{n, m}$ is $T N, B:=A[\alpha \mid \beta]$ is a contiguous, rank deficient submatrix of $A$, and both $A[1, \ldots, n \mid \beta]$ and $A[\alpha \mid 1, \ldots, m]$ have greater rank than $B$. Then either the left shadow or the right shadow of $B$ has the same rank as $B$.

Lemma 2.3. (E.g., [15, Theorem 1.13]) All principal minors of an NsTN matrix are positive.

Monotonicity properties of the determinant through matrix intervals are given in the next two lemmas.

Lemma 2.4. ([2, Lemma 3.2]) Let $[A, B] \in \mathbb{I}\left(\mathbb{R}^{n, n}\right), A$ be $N s T N$, and $B$ be $T N$. Then for any $Z \in[A, B]$, the following inequalities hold:

$$
\operatorname{det} A \leq \operatorname{det} Z \leq \operatorname{det} B \text {. }
$$

Lemma 2.5. Let $[A, B] \in \mathbb{I}\left(\mathbb{R}^{n, n}\right), A$ and $B$ be $T N$, and $A[2, \ldots, n]$ be nonsingular. Then for any $Z \in[A, B]$, the following inequalities are true:

$$
\frac{\operatorname{det} A}{\operatorname{det} A[2, \ldots, n]} \leq \frac{\operatorname{det} Z}{\operatorname{det} Z[2, \ldots, n]} \leq \frac{\operatorname{det} B}{\operatorname{det} B[2, \ldots, n]} .
$$

Proof. Put $A_{1}:=A+\epsilon E_{11}, Z_{1}:=Z+\epsilon E_{11}$, and $B_{1}:=B+\epsilon E_{11}$ for some $\epsilon>0$. Then $A_{1} \leq * Z_{1} \leq^{*} B_{1}$, $A_{1}$ is $N s T N$ since $A[2, \ldots, n]$ is nonsingular, and $B_{1}$ is $T N$. By [2, Lemma 3.2],

$$
\frac{\operatorname{det} A_{1}}{\operatorname{det} A_{1}[2, \ldots, n]} \leq \frac{\operatorname{det} Z_{1}}{\operatorname{det} Z_{1}[2, \ldots, n]} \leq \frac{\operatorname{det} B_{1}}{\operatorname{det} B_{1}[2, \ldots, n]} \text {. }
$$

By Laplace expansion along the first row of $A_{1}$, we obtain $\operatorname{det} A_{1}=\operatorname{det} A+\epsilon \operatorname{det} A[2, \ldots, n]$, with similar expansions of $\operatorname{det} Z_{1}$, and $\operatorname{det} B_{1}$, which we substitute into (2.1) to get

$$
\frac{\operatorname{det} A}{\operatorname{det} A[2, \ldots, n]}+\epsilon \leq \frac{\operatorname{det} Z}{\operatorname{det} Z[2, \ldots, n]}+\epsilon \leq \frac{\operatorname{det} B}{\operatorname{det} B[2, \ldots, n]}+\epsilon,
$$

from which the claim follows.

Finally, we recall a certain type of rank conditions associated with the rank of sets of submatrices of a matrix.

Definition 2.6. Let $A \in \mathbb{R}^{n, n}$. Then $A$ satisfies the descending rank conditions if for all $l$ with $1 \leq$ $l \leq n-1$, for all $z$ with $0 \leq z \leq l-1$, and for all $p$ with $l-z \leq p \leq n-z-1$, the following two sets of inequalities are satisfied:

$$
\begin{aligned}
& \operatorname{rank} A[p+1, \ldots, p+z+1 \mid 1, \ldots, l] \leq \operatorname{rank} A[p, \ldots, p+z \mid 1, \ldots, l] \\
& \operatorname{rank} A[1, \ldots, l \mid p+1, \ldots, p+z+1] \leq \operatorname{rank} A[1, \ldots, l \mid p, \ldots, p+z] .
\end{aligned}
$$




\section{The condensed form of the Cauchon Algorithm and some of its properties.}

3.1. The condensed form of the Cauchon Algorithm. We recall the definition of Cauchon diagrams and from [4] the condensed form of the Cauchon Algorithm which reduces the complexity of the orginal algorithm [12], [14].

In order to formulate the Cauchon Algorithm, we need the following notation. We denote by $\leq$ and $\leq_{c}$ the lexicographic and colexicographic orders, respectively, on $\mathbb{N}^{2}$, i.e.,

$$
\begin{gathered}
(g, h) \leq(i, j): \Leftrightarrow \quad(g<i) \text { or }(g=i \text { and } h \leq j), \\
(g, h) \leq_{c}(i, j): \Leftrightarrow \quad(h<j) \text { or }(h=j \text { and } g \leq i) .
\end{gathered}
$$

Definition 3.1. An $n$-by-m Cauchon diagram $C$ is an $n$-by- $m$ grid consisting of $n \cdot m$ squares colored black and white, where each black square has the property that either every square to its left (in the same row) or every square above it (in the same column) is black.

We denote by $\mathcal{C}_{n, m}$ the set of all $n$-by-m Cauchon diagrams. We fix positions in a Cauchon diagram in the following way: For $C \in \mathcal{C}_{n, m}$ and $i \in\{1, \ldots, n\}, j \in\{1, \ldots, m\},(i, j) \in C$ if the square in row $i$ and column $j$ is black. Here we use the usual matrix notation for the $(i, j)$ position in a Cauchon diagram, i.e., the square in the $(1,1)$ position of the Cauchon diagram is in its top left corner.

Definition 3.2. Let $A \in \mathbb{R}^{n, m}$ and let $C \in \mathcal{C}_{n, m}$. We say that $A$ is a Cauchon matrix associated with the Cauchon diagram $C$ if for all $(i, j), i \in\{1, \ldots, n\}, j \in\{1, \ldots, m\}$, we have $a_{i j}=0$ if and only if $(i, j) \in C$. If $A$ is a Cauchon matrix associated with an unspecified Cauchon diagram, we just say that $A$ is a Cauchon matrix.

Algorithm 1 (Condensed form of the Cauchon Algorithm) [1, Algorithm 3.3], [4, Algorithm 3.2]

Let $A=\left(a_{i j}\right) \in \mathbb{R}^{n, m}$. Set $A^{(n)}:=A$.

For $k=n-1, \ldots, 1$ define $A^{(k)}=\left(a_{i j}^{(k)}\right) \in \mathbb{R}^{n, m}$ as follows:

For $j=1, \ldots, m-1$,

set $s_{j}:=\min \left\{h \in\{j+1, \ldots, m\} \mid a_{k+1, h}^{(k+1)} \neq 0\right\}$ (set $s_{j}:=\infty$ if this set is empty), for $i=1, \ldots, k$,

$$
a_{i j}^{(k)}:= \begin{cases}a_{i j}^{(k+1)}-\frac{a_{k+1, j}^{(k+1)} a_{i, s_{j}}^{(k+1)}}{a_{k+1, s_{j}}^{(k+1)}}, & \text { if } s_{j}<\infty, \\ a_{i j}^{(k+1)}, & \text { if } s_{j}=\infty,\end{cases}
$$

and for $i=k+1, \ldots, n, j=1, \ldots, m$, and $i=1, \ldots, k, j=m$

$$
a_{i j}^{(k)}:=a_{i j}^{(k+1)}
$$

Put $\tilde{A}:=A^{(1)}$.

We conclude this subsection with two results on the application of the Cauchon Algorithm, see Algorithm 1 , to $T N$ matrices.

Theorem 3.3. ([12, Theorem B4] and [14, Theorem 2.6]) Let $A \in \mathbb{R}^{n, m}$. Then $A$ is $T N$ if and only if $\tilde{A}$ is an (entry-wise) nonnegative Cauchon matrix. 
3.2. $T N$ cells. For $\mathbb{R}^{n, m}$, fix a set $\mathcal{F}$ of minors. The $T N$ cell corresponding to the set $\mathcal{F}$ is the set of the $n$-by-m $T N$ matrices for which all their zero minors are just the ones from $\mathcal{F}$. In [14], it is proved that the Cauchon Algorithm provides a bijection between the nonempty $T N$ cells of $\mathbb{R}^{n, m}$ and $\mathcal{C}_{n, m}$. The following theorem gives more details about this mapping.

Theorem 3.4. ([14, Theorem 2.7])

(i) Let $A, B \in \mathbb{R}^{n, m}$ be $T N$. Then $A, B$ belong to the same $T N$ cell if and only if $\tilde{A}, \tilde{B}$ are associated with the same Cauchon diagram.

(ii) Let $A \in \mathbb{R}^{n, m}$. Then $A$ is contained in the $T N$ cell associated with $C \in \mathcal{C}_{n, m}$ if and only if $\tilde{a}_{i j}=0$ if $(i, j) \in C$ and $\tilde{a}_{i j}>0$ if $(i, j) \notin C$.

3.3. Lacunary sequences. We recall from [14] the definition of a lacunary sequence associated with a Cauchon diagram.

Definition 3.5. Let $C \in \mathcal{C}_{n, m}$. We say that a sequence

$$
\gamma:=\left(\left(i_{k}, j_{k}\right), \quad k=0,1, \ldots, t\right)
$$

which is strictly increasing in both arguments, is a lacunary sequence with respect to $C$ if the following conditions hold:

1. $\left(i_{k}, j_{k}\right) \notin C, k=1, \ldots, t$.

2. $(i, j) \in C$ for $i_{t}<i \leq n$ and $j_{t}<j \leq m$.

3. Let $s \in\{1, \ldots, t-1\}$. Then $(i, j) \in C$ if

(a) either for all $(i, j), i_{s}<i<i_{s+1}$ and $j_{s}<j$, or for all $(i, j), i_{s}<i<i_{s+1}$ and $j_{0} \leq j<j_{s+1}$ and

(b) either for all $(i, j), i_{s}<i$ and $j_{s}<j<j_{s+1}$ or for all $(i, j), i<i_{s+1}$, and $j_{s}<j<j_{s+1}$.

We call $t$ the length of $\gamma$.

We recall now from [4] and [8] the construction of two special lacunary sequences. In the first case, let $\delta_{i j}:=\operatorname{det} A\left[i_{0}, i_{1}, \ldots, i_{p} \mid j_{0}, j_{1}, \ldots, j_{p}\right]$ be the minor of $A$ associated to the sequence $\gamma$ given by (3.2) starting at position $(i, j)=\left(i_{0}, j_{0}\right)$ which is formed by the following procedure. We explain the construction only from the starting pair to the next index pair. The process is then continued analogously.

Procedure 3.6. ([4, Procedure 5.2]) Construction of the sequence $\gamma$ given by (3.2) starting at $\left(i_{0}, j_{0}\right)$ to the next index pair $\left(i_{1}, j_{1}\right)$ for the $n$-by-m TN matrix $A$.

$$
\begin{aligned}
& \text { If } i_{0}=n \text { or } j_{0}=m \text { or } \mathcal{U}:=\left\{(i, j) \mid i_{0}<i \leq n, j_{0}<j \leq m \text {, and } 0<\delta_{i j}\right\} \text { is void then terminate } \\
& \text { with } p:=0 ; \\
& \text { else } \quad \text { if } \delta_{i j_{0}}=0 \text { for all } i=i_{0}+1, \ldots, n \text { then } \text { put }\left(i_{1}, j_{1}\right):=\min \mathcal{U} \text { with } \\
& \quad \text { respect to the colexicographic order } \\
& \text { else } \\
& \text { put } i^{\prime}:=\min \left\{k \mid i_{0}<k \leq n \text { such that } 0<\delta_{k j_{0}}\right\} \\
& \qquad J:=\left\{k \mid j_{0}<k \leq m \text { such that } 0<\delta_{i^{\prime}, k}\right\}
\end{aligned}
$$




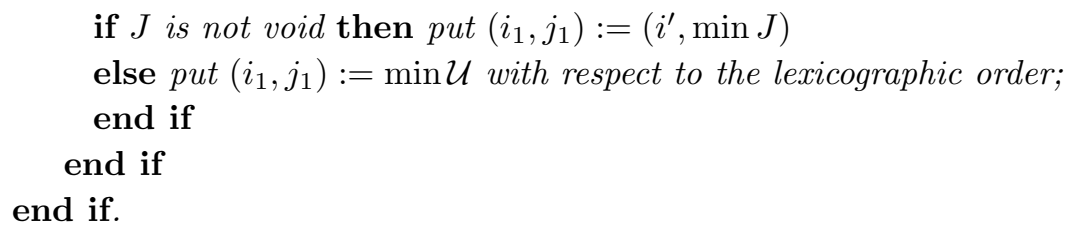

The following proposition provides a representation of the determinant of the submatrix associated to a lacunary sequence with respect to $C_{\tilde{A}}$.

Proposition 3.7. ([8, Corollary 3.3]) Let $A \in \mathbb{R}^{n, m}$ be such that $\tilde{A}$ is a Cauchon matrix and let $\gamma=$ $\left(\left(i_{0}, j_{0}\right),\left(i_{1}, j_{1}\right), \ldots,\left(i_{t}, j_{t}\right)\right)$ be a lacunary sequence with respect to $C_{\tilde{A}}$. Then the representation

$$
\operatorname{det} A\left[i_{0}, i_{1}, \ldots, i_{t} \mid j_{0}, j_{1}, \ldots, j_{t}\right]=\tilde{a}_{i_{0}, j_{0}} \cdot \tilde{a}_{i_{1}, j_{1}} \cdots \tilde{a}_{i_{t}, j_{t}}
$$

holds.

The following proposition shows that a certain sequence of zeros in a column or a row of $\tilde{A}$ is the result of a zero column or row or submatrix in the bottom left or top right part of $A$.

Proposition 3.8. Let $A \in \mathbb{R}^{n, m}$ be such that $\tilde{A} \in \mathbb{R}^{n, m}$ is a Cauchon matrix.

(i) If $\tilde{A}[i, \ldots, n \mid j]=0$ for some $i \in\{1, \ldots, n\}$ and $j \in\{1, \ldots, m\}$, then all entries of $A[i, \ldots, n \mid$ $1, \ldots, j]$ are zero or the $j$ th column of $A$ is zero.

(ii) If $\tilde{A}[i \mid j, \ldots, m]=0$ for some $i \in\{1, \ldots, n\}$ and $j \in\{1, \ldots, m\}$, then all entries of $A[1, \ldots, i \mid$ $j, \ldots, m]$ are zero or the ith row of $A$ is zero.

Proof. We only give the proof for (i) since the proof of (ii) is parallel. Since $\tilde{A}$ is a Cauchon matrix and $\tilde{A}[i, \ldots, n \mid j]=0$, we have $\tilde{A}[i, \ldots, n \mid 1, \ldots, j]=0$ or $\tilde{A}[1, \ldots, n \mid j]=0$. In the following we assume that $\tilde{A}[i, \ldots, n \mid 1, \ldots, j]=0$. We proceed by decreasing induction on the row index to show that $a_{s t}=0$, $s=i, \ldots, n, t=1, \ldots, j$. For $s=n$, by Algorithm $1, a_{n t}=\tilde{a}_{n t}=0, t=1, \ldots, j$. Assume that $a_{h t}=0$, $h=s+1, \ldots, n, t=1, \ldots, j$. We show that $a_{s t}=0, t=1, \ldots, j$. From each position $(s, t), t=1, \ldots, j$, we construct by Procedure 3.6 a lacunary sequence $\gamma_{s t}=\left((s, t),\left(s_{1}, t_{1}\right), \ldots,\left(s_{p}, t_{p}\right)\right)$ with respect to $C_{\tilde{A}}$. If $\gamma_{s t}=((s, t))$, then by Proposition 3.7,

$$
a_{s t}=\operatorname{det} A[s \mid t]=\tilde{a}_{s t}=0
$$

Therefore, we assume in the following that $\gamma_{s t}$ has positive length. By the induction hypothesis and Laplace expansion along the first column of $A\left[s, s_{1}, \ldots, s_{p} \mid t, t_{1}, \ldots, t_{p}\right]$, we obtain

$$
\operatorname{det} A\left[s, s_{1}, \ldots, s_{p} \mid t, t_{1}, \ldots, t_{p}\right]=a_{s t} \operatorname{det} A\left[s_{1}, \ldots, s_{p} \mid t_{1}, \ldots, t_{p}\right]
$$

Since $\gamma_{s t}$ and $\left(\left(s_{1}, t_{1}\right), \ldots,\left(s_{p}, t_{p}\right)\right)$ are lacunary sequences, it follows from Proposition 3.7 that

$$
\begin{aligned}
\operatorname{det} A\left[s, s_{1}, \ldots, s_{p} \mid t, t_{1}, \ldots, t_{p}\right] & =\tilde{a}_{s t} \cdot \tilde{a}_{s_{1}, t_{1}} \cdots \tilde{a}_{s_{p}, t_{p}} \\
& =0 \cdot \operatorname{det} A\left[s_{1}, \ldots, s_{p} \mid t_{1}, \ldots, t_{p}\right] .
\end{aligned}
$$

Moreover, $\operatorname{det} A\left[s_{1}, \ldots, s_{p} \mid t_{1}, \ldots, t_{p}\right] \neq 0$ since $\left(\left(s_{1}, t_{1}\right), \ldots,\left(s_{p}, t_{p}\right)\right)$ is a lacunary sequence that starts from a nonzero entry. Therefore, we conclude from (3.4) that $a_{s t}=0$. Since $t \in\{1, \ldots, j\}$ was chosen arbitrarily, we conclude that $A[i, \ldots, n \mid 1, \ldots, j]=0$. If the $j$ th column of $\tilde{A}$ is zero we proceed as above to show that then also the $j$ th column of $A$ is zero, which completes the proof. 
Let $A \in \mathbb{R}^{n, m}$ be such that $\tilde{A}$ is a Cauchon matrix. Then by the following procedure, a uniquely determined lacunary sequence is constructed which is related to the rank of $A$.

Procedure 3.9. Let $\tilde{A} \in \mathbb{R}^{n, m}$ be a Cauchon matrix. Construct the sequence

$$
\gamma=\left(\left(i_{p}, j_{p}\right), \ldots,\left(i_{0}, j_{0}\right)\right)
$$

as follows:

- Put $\left(i_{-1}, j_{-1}\right):=(n+1, m+1)$.

- For $k=0,1, \ldots$, define

$$
M_{k}:=\left\{(i, j) \mid 1 \leq i<i_{k-1}, \quad 1 \leq j<j_{k-1}, \tilde{a}_{i j} \neq 0\right\}
$$

If $M_{k}=\phi$, put $p:=k-1$. Otherwise, put $\left(i_{k}, j_{k}\right):=\max M_{k}$, where the maximum is taken with respect to the lexicographic order.

Proposition 3.10. Let $A \in \mathbb{R}^{n, m}$ be such that $\tilde{A}$ is a Cauchon matrix. Then for all $(i, j) \in \mathcal{S}$,

$$
\operatorname{rank}(A[i, i+1, \ldots, n \mid 1,2, \ldots, j])=\eta+1,
$$

where $\eta$ is the length of the sequence that is obtained by application of Procedure 3.9 to $\tilde{A}[i, i+1, \ldots, n \mid$ $1,2, \ldots, j]$, provided that $A[i, i+1, \ldots, n \mid 1,2, \ldots, j] \neq 0$.

Proof. The matrix that is obtained by application of Algorithm 1 to $B:=A[i, i+1, \ldots, n \mid 1,2, \ldots, m]$ coincides with $\tilde{A}[i, i+1, \ldots, n \mid 1,2, \ldots, m]$. Hence, if we apply Procedure 3.9 to $\tilde{B}[1, \ldots, n-i+1 \mid 1, \ldots, j]=$ $\tilde{A}[i, i+1, \ldots, n \mid 1,2, \ldots, j]$ and proceed parallel to the proof of [8, Theorem 3.4], we are done.

Corollary 3.11. Let $A \in \mathbb{R}^{n, m}$ be such that $\tilde{A}$ is a Cauchon matrix. Then for all $(i, j) \in \mathcal{S}$,

$$
\operatorname{rank}(A[1,2, \ldots, i \mid j, j+1, \ldots, m])=\eta+1
$$

where $\eta$ is the length of the sequence that is obtained by application of Procedure 3.9 to $\tilde{A}[1,2, \ldots, i \mid j, j+$ $1, \ldots, m]$, provided that $A[1,2, \ldots, i \mid j, j+1, \ldots, m] \neq 0$.

Theorem 3.12. ([8, Theorem 3.2]) Let $A \in \mathbb{R}^{n, m}$ be such that $\tilde{A}$ is a Cauchon matrix. Then for $i=1, \ldots, n$ and $0 \leq l \leq n-i$, the rows $i, i+1, \ldots, i+l$ of $A$ are linearly independent if and only if application of Procedure 3.9 to $\tilde{A}[i, \ldots, i+l \mid 1, \ldots, m]$ results in a sequence of length $l$.

Corollary 3.13. ([8, Corollary 3.2]) Let $A \in \mathbb{R}^{n, m}$ be such that $\tilde{A}$ is a Cauchon matrix. Then for $j=1, \ldots, m$ and $0 \leq l \leq m-j$, the columns $j, j+1, \ldots, j+l$ of $A$ are linearly independent if and only if application of Procedure 3.9 to $\tilde{A}[1, \ldots, n \mid j, \ldots, j+l]$ results in a sequence of length $l$.

3.4. Descending rank conditions. In this subsection, we link the descending rank conditions, see Definition 2.6, to Algorithm 1.

Theorem 3.14. ([8, Theorem 4.4]) Let $A \in \mathbb{R}^{n, n}$ and $B:=A^{\#}$. If $A$ satisfies the descending rank conditions, then the following statements hold:

(i) If $\tilde{b}_{i j}=0$ for some $i \geq j$, then $\tilde{b}_{i t}=0$ for all $t<j$.

(ii) If $\tilde{b}_{i j}=0$ for some $i \leq j$, then $\tilde{b}_{t j}=0$ for all $t<i$.

(iii) $\tilde{B}$ is a Cauchon matrix. 
TheOREm 3.15. ([8, Theorem 4.8]) Let $A \in \mathbb{R}^{n, n}$ and $B:=A^{\#}$. Then the following statements are equivalent:

(a) A satisfies the descending rank conditions.

(b) B satisfies (i) and (ii) in Theorem 3.14.

4. Relaxing nonsingularity to linear independence of certain rows and columns. For the rest of the paper, we assume for the ease of presentation that the given $T N$ matrices do not contain a zero row or column. This is not a restriction because after deletion of the respective rows and columns the resulting matrix is again $T N$.

Definition 4.1. Let $A \in \mathbb{R}^{n, m}$ be such that $\tilde{A}$ is a Cauchon matrix. For a given lacunary sequence $\gamma=\left(\left(i_{0}, j_{0}\right),\left(i_{1}, j_{1}\right), \ldots,\left(i_{p}, j_{p}\right)\right)$, the order of the sequence is given by

$$
l:=\min \left\{k \mid \tilde{A}\left[i_{k}+1, \ldots, n \mid j_{k}\right]=0 \text { or } \tilde{A}\left[i_{k} \mid j_{k}+1, \ldots, m\right]=0\right\}
$$

we set $l:=p$ if the set in (4.6) is empty.

Condition I. Let $A \in \mathbb{R}^{n, m}$ be such that $\tilde{A}$ is a Cauchon matrix. For all $(i, j) \in \mathcal{S}$, the rows $i+1, \ldots, i+\ell$ and columns $j+1, \ldots, j+\ell$ of $A$ are linearly independent provided that $\ell>0$, where $\ell$ is the smallest among the orders of all the lacunary sequences with respect to $C_{\tilde{A}}$ that start from $(i, j)$.

In the sequel, it will always be clear from the context to which pairs $(i, j) \in \mathcal{S}$ the quantity $\ell$ refers. Therefore, it will not be necessary to indicate this dependency.

LEMma 4.2. Let $A \in \mathbb{R}^{n, m}$ be such that $\tilde{A}$ is a Cauchon matrix and assume that Condition I holds. Then for any $(i, j) \in \mathcal{S}$ with $\ell>0$, there exists a lacunary sequence $\gamma=\left((i, j),\left(i_{1}, j_{1}\right), \ldots,\left(i_{p}, j_{p}\right)\right)$ with respect to $C_{\tilde{A}}$ of order $\ell$ starting from $(i, j)$ such that

$$
d\left(i, i_{1}, \ldots, i_{\ell}\right)=0 \quad \text { or } \quad d\left(j, j_{1}, \ldots, j_{\ell}\right)=0,
$$

where $\ell$ is given as in Condition $I$.

Proof. Suppose on the contrary that there exists $\left(i_{0}, j_{0}\right) \in \mathcal{S}$ with $\ell>0$ such that for any lacunary sequence $\gamma=\left(\left(i_{0}, j_{0}\right),\left(i_{1}, j_{1}\right), \ldots,\left(i_{p}, j_{p}\right)\right)$ with respect to $C_{\tilde{A}}$ of order $\ell$ we have $d\left(i_{0}, i_{1}, \ldots, i_{\ell}\right)>0$ and $d\left(j_{0}, j_{1}, \ldots, j_{\ell}\right)>0$. Moreover, assume that $\gamma$ is chosen in such a way that $\left(i_{0}, j_{0}\right)$ is the maximum such pair with respect to the lexicographic order. Therefore, we may conclude that

$$
d\left(i_{1}, \ldots, i_{\ell}\right)=0 \quad \text { or } \quad d\left(j_{1}, \ldots, j_{\ell}\right)=0
$$

Without loss of generality we may assume that $d\left(j_{1}, \ldots, j_{\ell}\right)=0$ and $j_{1}=j_{0}+2$.

Case 1: $i_{\ell}=n$ or $\tilde{a}_{s, j_{\ell}}=0, s=i_{\ell}+1, \ldots, n$. If $\tilde{a}_{s, j_{\ell}}=0, s=i_{\ell}+1, \ldots, n$, then it follows that $\tilde{A}\left[i_{\ell}+1, \ldots, n \mid 1, \ldots, j_{\ell}\right]=0$ because $\tilde{A}$ is a Cauchon matrix. Hence, in either case it is easy to see that $\left(i_{\ell}, j_{\ell}\right)$ is the maximum pair with respect to the lexicographic order of the set

$$
\left\{(u, v) \mid 1 \leq u \leq n, 1 \leq v \leq j_{\ell}, \tilde{a}_{u v} \neq 0\right\}
$$

Moreover, since $d\left(j_{1}, \ldots, j_{\ell}\right)=0$ and $\gamma=\left((i, j),\left(i_{1}, j_{1}\right), \ldots,\left(i_{p}, j_{p}\right)\right)$ is a lacunary sequence with respect to $C_{\tilde{A}}$, for $s=1, \ldots, \ell-1$, we have $\left(i_{s}, j_{s}\right)$ is the maximum pair with respect to the lexicographic order of the set

$$
\left\{(u, v) \mid 1 \leq u<i_{s+1}, 1 \leq v<j_{s+1}, \tilde{a}_{u v} \neq 0\right\}
$$


Therefore, the sequence which is obtained by the application of Procedure 3.9 to the columns $j_{1}, j_{2}, \ldots, j_{\ell}$ coincides with the sequence $\left(\left(i_{1}, j_{1}\right),\left(i_{2}, j_{2}\right), \ldots,\left(i_{\ell}, j_{\ell}\right)\right)$. Now we apply Procedure 3.9 to the columns $j_{0}+$ $1, j_{0}+2, \ldots, j_{0}+\ell$ which coincide with the columns $j_{0}+1=j_{1}-1, j_{2}-1, \ldots, j_{\ell}-1$. This results in the lacunary sequence $\left(\left(i_{1}^{\prime}, j_{1}^{\prime}\right), \ldots,\left(i_{\tau}^{\prime}, j_{\tau}^{\prime}\right)\right)$, where $\tau \leq \ell$. If $\tau \leq \ell-1$, then by Corollary 3.13, the columns $j_{0}+1, j_{0}+2, \ldots, j_{0}+\ell$ are linearly dependent which contradicts Condition I. Therefore, we have $\tau=\ell$, and hence, $j_{k}^{\prime}=j_{k}-1, k=1,2, \ldots, \ell=\tau$. Since $\gamma$ is a lacunary sequence, $\ell \geq 1, A$ does not have a zero row or column, and $j_{1}=j_{0}+2$, we have

$$
\tilde{a}_{t, j_{0}+1}=0, \quad t=1,2, \ldots, i_{1}-1,
$$

which implies that $i_{1}^{\prime}>i_{0}$. Since application of Procedure 3.9 to the columns $j_{1}, j_{2}, \ldots, j_{\ell}$ results in the sequence $\left(\left(i_{1}, j_{1}\right),\left(i_{2}, j_{2}\right), \ldots,\left(i_{\ell}, j_{\ell}\right)\right)$ and $d\left(j_{1}, \ldots, j_{\ell}\right)=0$, we conclude that for $g=0,1, \ldots, \ell-1$, if $d\left(i_{g}, i_{g+1}\right)>0$, then it follows that $\tilde{a}_{u v}=0, u=i_{g}+1, \ldots, i_{g+1}-1, v=1, \ldots, i_{g+1}-1$. Therefore, we may conclude that

$$
i_{k}^{\prime}=i_{k}, \quad k=1,2, \ldots, \ell=\tau .
$$

Hence, the sequence which is obtained by appending $\left(\left(i_{0}, j_{0}\right),\left(i_{1}^{\prime}, j_{1}^{\prime}\right), \ldots,\left(i_{\ell}^{\prime}, j_{\ell}^{\prime}\right)\right)$ to a lacunary sequence which starts from $\left(i_{\ell}^{\prime}, j_{\ell}^{\prime}\right)$ is a lacunary sequence with respect to $C_{\tilde{A}}$, has order $\ell$, and $d\left(j_{0}, j_{1}^{\prime}, \ldots, j_{\ell}^{\prime}\right)=0$ which contradicts our assumption.

Case 2: $j_{\ell}=m$ or $\tilde{a}_{i_{\ell}, s}=0, s=j_{\ell}+1, \ldots, m$. The proof is parallel to the one of Case 1 .

Lemma 4.3. Let $A \in \mathbb{R}^{n, m}$ be $T N$ and suppose Condition I holds. Then for any $(i, j) \in \mathcal{S}$ with $\ell>0$ we have

$$
\operatorname{det} A[i+1, i+2, \ldots, i+\ell \mid j+1, j+2, \ldots, j+\ell]>0,
$$

where $\ell$ is given as in Condition $I$.

Proof. By Theorem 3.3, $\tilde{A}$ is a Cauchon matrix. Suppose on the contrary that there exists $\left(i_{0}, j_{0}\right) \in \mathcal{S}$ such that the determinant of the matrix

$$
B:=A\left[i_{0}+1, i_{0}+2, \ldots, i_{0}+\ell \mid j_{0}+1, j_{0}+2, \ldots, j_{0}+\ell\right]
$$

vanishes. Moreover, assume that $\left(i_{0}, j_{0}\right)$ is the maximum such pair with respect to the lexicographic order and let $\gamma=\left(\left(i_{0}, j_{0}\right),\left(i_{1}, j_{1}\right), \ldots,\left(i_{p}, j_{p}\right)\right)$ be an associated lacunary sequence with respect to $C_{\tilde{A}}$ of order $\ell>0$ with $d\left(i_{0}, i_{1}, \ldots, i_{\ell}\right)=0$ or $d\left(j_{0}, j_{1}, \ldots, j_{\ell}\right)=0$ which exists by Lemma 4.2 . Without loss of generality, we may assume that $d\left(j_{0}, j_{1}, \ldots, j_{\ell}\right)=0$. By Lemma 2.2 and Condition I, the left or the right shadow of $B$ has rank at most $\ell-1$. Since $\left(\left(i_{1}, j_{1}\right), \ldots,\left(i_{p}, j_{p}\right)\right)$ is a lacunary sequence with $\tilde{a}_{i_{1}, j_{1}} \neq 0$, we have by Proposition 3.7

$$
\operatorname{det} A\left[i_{1}, \ldots, i_{p} \mid j_{1}, \ldots, j_{p}\right] \neq 0,
$$

and we conclude by Lemma 2.3 that

$$
\operatorname{det} A\left[i_{1}, \ldots, i_{\ell} \mid j_{1}, \ldots, j_{\ell}\right] \neq 0 .
$$

Because $A\left[i_{1}, \ldots, i_{\ell} \mid j_{1}, \ldots, j_{\ell}\right]$ lies completely in the left shadow of $B$, the left shadow of $B$ has rank at least $\ell$. By Theorem 3.12, application of Procedure 3.9 to the rows $i_{0}+1, \ldots, i_{0}+\ell$ results in the lacunary sequence $\left(\left(i_{0}+1, \beta_{1}\right),\left(i_{0}+2, \beta_{2}\right), \ldots,\left(i_{0}+\ell, \beta_{\ell}\right)\right)$. If $\beta_{1}>j_{0}$, then by Corollary 3.11 , the right shadow of $A\left[i_{0}+1, i_{0}+2, \ldots, i_{0}+\ell \mid j_{0}+1, j_{0}+2, \ldots, j_{0}+\ell\right]$ has rank at least $\ell$. Now we assume that $\beta_{1} \leq j_{0}$. Let $s \in\{1,2, \ldots, \ell\}$ be the smallest integer such that $\beta_{s}>j_{0}$. Note that $s \geq 2$. Define $\left(i_{0}^{\prime}, j_{0}^{\prime}\right)=\left(i_{0}, j_{0}\right)$ and for $k=1,2, \ldots, \tau$, let

$$
\left(i_{k}^{\prime}, j_{k}^{\prime}\right):=\min \left\{(i, j) \mid i=i_{k-1}^{\prime}+1, \quad j>j_{k-1}, \quad \tilde{a}_{i j}>0\right\},
$$


where the minimum is taken with respect to the lexicographic order. Consider the sequence $\left(\left(i_{0}^{\prime}, j_{0}^{\prime}\right),\left(i_{1}^{\prime}, j_{1}^{\prime}\right)\right.$, $\left.\ldots,\left(i_{\tau}^{\prime}, j_{\tau}^{\prime}\right)\right)$. If $j_{\tau}^{\prime}=m$, then this sequence is a lacunary sequence with respect to $C_{\tilde{A}}$ since for each $t=0,1, \ldots, \tau-1, i_{t+1}^{\prime}=i_{t}^{\prime}+1$ and there exists $\xi_{t+1}<j_{t+1}^{\prime}$ such that $\tilde{a}_{i_{t+1}^{\prime}, \xi_{t+1}}>0$. Otherwise, we append it to a lacunary sequence starting from $\left(i_{\tau}^{\prime}, j_{\tau}^{\prime}\right)$ such that the resulting sequence is a lacunary sequence with respect to $C_{\tilde{A}}$. Hence, the order of this sequence is $\tau$ which is less than $\ell$ and $d\left(i_{0}^{\prime}, i_{1}^{\prime}, \ldots, i_{\tau}^{\prime}\right)=0$ which contradicts our assumption. Therefore, $\beta_{1}>j_{0}$ and the right shadow of $B$ has rank at least $\ell$ which implies by Lemma 2.2 that $\operatorname{det} B>0$, a contradiction. Since we have obtained a contradiction both in the event of a left and right shadow, the proof is completed.

Now we turn to the construction of a lacunary sequence with the properties stated in Lemma 4.2. The procedure is based on the following lemma.

Lemma 4.4. Let $A \in \mathbb{R}^{n, m}$ be such that $\tilde{A}$ is a Cauchon matrix and suppose Condition I holds. Then for all $(i, j) \in \mathcal{S}$ such that $\tilde{A}[i+1, \ldots, n \mid j+1, \ldots, m] \neq 0$, let

$$
\begin{aligned}
s_{j} & :=\min \left\{k \in\{i+1, \ldots, n\} \mid \tilde{a}_{k j} \neq 0\right\}, \\
t_{i} & :=\min \left\{k \in\{j+1, \ldots, m\} \mid \tilde{a}_{i k} \neq 0\right\},
\end{aligned}
$$

provided that both sets are not empty. Then it follows that

$$
\tilde{a}_{s_{j}, j+1} \neq 0 \text { or } \quad \tilde{a}_{i+1, t_{i}} \neq 0 .
$$

Proof. Suppose on the contrary that there exists $\left(i_{0}, j_{0}\right) \in \mathcal{S}$ such that $\tilde{A}\left[i_{0}+1, \ldots, n \mid j_{0}+1, \ldots, m\right] \neq 0$ and $\tilde{a}_{s_{j_{0}}, j_{0}+1}=0$ and $\tilde{a}_{i_{0}+1, t_{i}}=0$. Hence, $\tilde{A}\left[i_{0}+1, i_{0}+2, \ldots, s_{j_{0}} \mid j_{0}+1, j_{0}+2, \ldots, t_{i_{0}}\right] \neq 0, \tilde{A}\left[i_{0}+1, i_{0}+\right.$ $\left.2, \ldots, s_{j_{0}} \mid j_{0}+1\right]=0$, and $\tilde{A}\left[i_{0}+1 \mid j_{0}+1, j_{0}+2, \ldots, t_{i_{0}}\right]=0$ since $\tilde{A}$ is a Cauchon matrix, $\tilde{a}_{s_{j_{0}}, j_{0}} \neq 0$, and $\tilde{a}_{i_{0}, t_{i}} \neq 0$. Therefore, for any lacunary sequence $\gamma=\left(\left(i_{0}, j_{0}\right),\left(i_{1}, j_{1}\right), \ldots,\left(i_{p}, j_{p}\right)\right)$ that starts from $\left(i_{0}, j_{0}\right)$ we have $d\left(i_{0}, i_{1}, \ldots, i_{\ell}\right)>0$ and $d\left(j_{0}, j_{1}, \ldots, j_{\ell}\right)>0$, where $\ell$ is the order of $\gamma$, which contradicts Lemma 4.2 .

Procedure 4.5. Construction of a lacunary sequence $\gamma=\left(\left(i_{0}, j_{0}\right),\left(i_{1}, j_{1}\right), \ldots,\left(i_{p}, j_{p}\right)\right)$ starting at $\left(i_{0}, j_{0}\right) \in \mathcal{S}$ to the next index pair $\left(i_{1}, j_{1}\right)$ in the $n$-by-m matrix $A$ such that $\tilde{A}$ is a Cauchon matrix and $A$ satisfies Condition $I$.

If $\mathcal{U}:=\left\{(i, j) \mid i_{0}<i \leq n, j_{0}<j \leq m\right.$, and $\left.0<\tilde{a}_{i, j}\right\}$ is void then terminate with $p:=0$; else

if $\tilde{a}_{i, j_{0}}=0$ for all $i=i_{0}+1, \ldots, n$ or $\tilde{a}_{i_{0}, j}=0$ for all $j=j_{0}+1, \ldots, m$

then put $\left(i_{1}, j_{1}\right):=\min \mathcal{U}$ with respect to the colexicographic order and lexicographic order, respectively;

else $p u t$

$$
\begin{aligned}
& i^{\prime}:=\min \left\{k \mid i_{0}<k \leq n \text { such that } \tilde{a}_{k, j_{0}} \neq 0\right\} \\
& j^{\prime}:=\min \left\{k \mid j_{0}<k \leq m \text { such that } \tilde{a}_{i_{0}, j} \neq 0\right\}
\end{aligned}
$$

if $\tilde{a}_{i^{\prime}, j_{0}+1} \neq 0$ then put $\left(i_{1}, j_{1}\right):=\left(i^{\prime}, j_{0}+1\right)$;

else put $\left(i_{1}, j_{1}\right):=\left(i_{0}+1, j^{\prime}\right)$;

end if

end if

end if. 
5. Application to intervals of totally nonnegative matrices. In this section, we consider matrices that satisfy Condition I. In [2], the proof of the interval property of the NsTN matrices relies on the fact that the entries of $\tilde{A}$ obtained from $A$ by application of Algorithm 1 can be represented as a ratio of contiguous minors of $A$. If we relax the nonsingularity assumption and would like to employ such a representation, we have to avoid division by a zero minor. We accomplish this by using Lemma 2.2, where the linear independence of the respective rows and columns is assured by Condition I. Then only the vanishing of the left or the right shadow of a zero contiguous minor has to be considered.

Let $A \in \mathbb{R}^{n, m}$ be $T N$. For any $\left(i_{0}, j_{0}\right) \in \mathcal{S}$, we can construct a lacunary sequence $\left(\left(i_{0}, j_{0}\right),\left(i_{1}, j_{1}\right)\right.$, $\left.\ldots,\left(i_{p}, j_{p}\right)\right)$ with respect to the Cauchon diagram $C_{\tilde{A}}$, and by Proposition 3.7 we may conclude that

$$
\operatorname{det} A\left[i_{0}, i_{1}, \ldots, i_{p} \mid j_{0}, j_{1}, \ldots, j_{p}\right]=\tilde{a}_{i_{0}, j_{0}} \cdot \tilde{a}_{i_{1}, j_{1}} \cdots \tilde{a}_{i_{p}, j_{p}} .
$$

Hence, by application of this representation to the lacunary sequence $\left(\left(i_{1}, j_{1}\right), \ldots,\left(i_{p}, j_{p}\right)\right)$, we obtain that

$$
\tilde{a}_{i_{0}, j_{0}}=\frac{\operatorname{det} A\left[i_{0}, i_{1}, \ldots, i_{p} \mid j_{0}, j_{1}, \ldots, j_{p}\right]}{\operatorname{det} A\left[i_{1}, \ldots, i_{p} \mid j_{1}, \ldots, j_{p}\right]} .
$$

Therefore, each entry of $\tilde{A}$ can be represented as a ratio of two minors. We want to strengthen this representation in that each entry of $\tilde{A}$ can even be represented as a ratio of two contiguous minors. We call $p$ the order of the representation (5.8).

Now let $A$ in addition satisfy Condition I with $\ell>0$. Then by Procedure 4.5 , for any $\left(i_{0}, j_{0}\right) \in \mathcal{S}$, we can construct a lacunary sequence $\left(\left(i_{0}, j_{0}\right),\left(i_{1}, j_{1}\right), \ldots,\left(i_{p}, j_{p}\right)\right)$ of order $\ell$ with (4.7). Without loss of generality, we may assume that $d\left(j_{0}, j_{1}, \ldots, j_{\ell}\right)=0$ holds. By Proposition $3.8, A\left[i_{\ell}+1, \ldots, n \mid 1, \ldots, j_{\ell}\right]=0$ or $A\left[1, \ldots, i_{\ell} \mid j_{\ell}+1, \ldots, m\right]=0$ holds. By (5.8) and the zero-nonzero pattern of $A$, we have

$$
\begin{aligned}
\tilde{a}_{i_{0}, j_{0}} & =\frac{\operatorname{det} A\left[i_{0}, i_{1}, \ldots, i_{p} \mid j_{0}, j_{1}, \ldots, j_{p}\right]}{\operatorname{det} A\left[i_{1}, \ldots, i_{p} \mid j_{1}, \ldots, j_{p}\right]} \\
& =\frac{\operatorname{det} A\left[i_{0}, i_{1}, \ldots, i_{\ell} \mid j_{0}, j_{1}, \ldots, j_{\ell}\right] \operatorname{det} A\left[i_{\ell+1}, \ldots, i_{p} \mid j_{\ell+1}, \ldots, j_{p}\right]}{\operatorname{det} A\left[i_{1}, \ldots, i_{\ell} \mid j_{1}, \ldots, j_{\ell}\right] \operatorname{det} A\left[i_{\ell+1}, \ldots, i_{p} \mid j_{\ell+1}, \ldots, j_{p}\right]} \\
& =\frac{\operatorname{det} A\left[i_{0}, i_{1}, \ldots, i_{\ell} \mid j_{0}, j_{1}, \ldots, j_{\ell}\right]}{\operatorname{det} A\left[i_{1}, \ldots, i_{\ell} \mid j_{1}, \ldots, j_{\ell}\right]} .
\end{aligned}
$$

Proposition 5.1. Let $A=\left(a_{i j}\right) \in \mathbb{R}^{n, m}$ be $T N$ and suppose Condition I holds. Then the entries $\tilde{a}_{i j}$ of the matrix $\tilde{A}$ can be represented as

$$
\tilde{a}_{i, j}=\frac{\operatorname{det} A[i, i+1, \ldots, i+\ell \mid j, j+1, \ldots, j+\ell]}{\operatorname{det} A[i+1, \ldots, i+\ell \mid j+1, \ldots, j+\ell]},
$$

where $\ell$ is given in Condition I and is assumed to be positive.

Proof. By Theorem 3.3, $\tilde{A}$ is a nonnegative Cauchon matrix. By the preceding consideration, for each position $\left(i_{0}, j_{0}\right) \in \mathcal{S}$, there exists a lacunary sequence $\left(\left(i_{0}, j_{0}\right),\left(i_{1}, j_{1}\right), \ldots,\left(i_{p}, j_{p}\right)\right)$ with respect to the Cauchon diagram $C_{\tilde{A}}$ of order $\ell$ such that

$$
\tilde{a}_{i_{0}, j_{0}}=\frac{\operatorname{det} A\left[i_{0}, i_{1}, \ldots, i_{\ell} \mid j_{0}, j_{1}, \ldots, j_{\ell}\right]}{\operatorname{det} A\left[i_{1}, \ldots, i_{\ell} \mid j_{1}, \ldots, j_{\ell}\right]} .
$$

Using Lemma 4.2, we can assume without loss of generality that $d\left(j_{0}, j_{1}, \ldots, j_{\ell}\right)=0$. By Proposition 3.7 and Lemma $2.3, \operatorname{det} A\left[i_{1}, i_{2}, \ldots, i_{\ell} \mid j_{1}, j_{2}, \ldots, j_{\ell}\right] \neq 0$ holds, since $\left(\left(i_{1}, j_{1}\right), \ldots,\left(i_{p}, j_{p}\right)\right)$ is a lacunary sequence 
and $\operatorname{det} A\left[i_{1}, i_{2}, \ldots, i_{p} \mid j_{1}, j_{2}, \ldots, j_{p}\right] \neq 0$. By Proposition 3.10, the rank of the matrix $B:=A\left[i_{0}+1, i_{0}+\right.$ $\left.2, \ldots, n \mid 1,2, \ldots, j_{\ell}\right]$ is $\ell$. Let $R_{i_{0}+1}, R_{i_{0}+2}, \ldots, R_{n}$ be the rows of the matrix $B$. Hence, we may represent $R_{h}=\sum_{s=1}^{\ell} \alpha_{h, s} R_{i_{s}}, h=i_{0}+1, i_{0}+2, \ldots, i_{0}+\ell$. Therefore, we may conclude

$$
A\left[i_{0}+1, i_{0}+2, \ldots, i_{0}+\ell \mid 1,2, \ldots, j_{\ell}\right]=C A\left[i_{1}, \ldots, i_{\ell} \mid 1,2, \ldots, j_{\ell}\right]
$$

where $C=\left(c_{t_{1}, t_{2}}\right) \in \mathbb{R}^{\ell, \ell}$ with $c_{t_{1}, t_{2}}=\alpha_{i_{0}+t_{1}, t_{2}}, t_{1}, t_{2}=1,2, \ldots, \ell$.

In particular, we obtain for a special choice of the column vectors

$$
\begin{aligned}
A\left[i_{0}+1, i_{0}+2, \ldots, i_{0}+\ell \mid j_{0}+1, j_{0}+2, \ldots, j_{0}+\ell\right] & =C A\left[i_{1}, i_{2}, \ldots, i_{\ell} \mid j_{0}+1, j_{0}+2, \ldots, j_{0}+\ell\right] \\
& =C A\left[i_{1}, i_{2}, \ldots, i_{\ell} \mid j_{1}, j_{2}, \ldots, j_{\ell}\right]
\end{aligned}
$$

whence

$$
\operatorname{det} A\left[i_{0}+1, i_{0}+2, \ldots, i_{0}+\ell \mid j_{0}+1, j_{0}+2, \ldots, j_{0}+\ell\right]=\operatorname{det} C \operatorname{det} A\left[i_{1}, i_{2}, \ldots, i_{\ell} \mid j_{1}, j_{2}, \ldots, j_{\ell}\right]
$$

Since by Lemma 4.3

$$
\operatorname{det} A\left[i_{0}+1, i_{0}+2, \ldots, i_{0}+\ell \mid j_{0}+1, j_{0}+2, \ldots, j_{0}+\ell\right] \neq 0
$$

and

$$
\operatorname{det} A\left[i_{1}, i_{2}, \ldots, i_{\ell} \mid j_{1}, j_{2}, \ldots, j_{\ell}\right] \neq 0
$$

we conclude that $\operatorname{det} C \neq 0$.

Moreover, we obtain

$$
A\left[i_{0}, i_{0}+1, \ldots, i_{0}+\ell \mid j_{0}, j_{0}+1, \ldots, j_{0}+\ell\right]=C^{\prime} A\left[i_{0}, i_{1}, \ldots, i_{\ell} \mid j_{0}, j_{1}, \ldots, j_{\ell}\right]
$$

where $C^{\prime} \in \mathbb{R}^{\ell+1, \ell+1}$ is given by

$$
C^{\prime}=\left[\begin{array}{ll}
1 & 0 \\
0 & C
\end{array}\right]
$$

which yields

$$
\operatorname{det} A\left[i_{0}, i_{0}+1, \ldots, i_{0}+\ell \mid j_{0}, j_{0}+1, \ldots, j_{0}+\ell\right]=\operatorname{det} C^{\prime} \operatorname{det} A\left[i_{0}, i_{1}, \ldots, i_{\ell} \mid j_{0}, j_{1}, \ldots, j_{\ell}\right]
$$

Since $\operatorname{det} C^{\prime}=\operatorname{det} C$, the representation follows now from (5.11)-(5.13) .

TheOREm 5.2. Let $A=\left(a_{k j}\right), B=\left(b_{k j}\right) \in \mathbb{R}^{n, m}$ be $T N$ such that Condition $I$ holds and $A \leq \leq^{*} B$. Then $\tilde{A} \leq^{*} \tilde{B}$ and the entries $\tilde{a}_{k j}$ and $\tilde{b}_{k j}$ of $\tilde{A}$ and $\tilde{B}$, respectively, can be represented as ratios of contiguous minors of the same order, $k=1, \ldots, n, j=1, \ldots, m$.

Proof. Let $A$ and $B$ be $T N$. Then by Theorem 3.3, $\tilde{A}$ and $\tilde{B}$ are nonnegative Cauchon matrices. We show by decreasing induction with respect to the lexicographic order on $(k, j)$ that if $\tilde{a}_{k j}$ and $\tilde{b}_{k j}$ have representations as in (5.10) of order $l$ and $l^{\prime}$, respectively, then both of them can be represented as ratios of contiguous minors of the same order and $(-1)^{k+j} \tilde{a}_{k j} \leq(-1)^{k+j} \tilde{b}_{k j}$. For $k=n$ or $j=m$, the result is trivial and follows by the application of Algorithm 1 and the assumption that $A \leq^{*} B$. Suppose the claim holds for all $\left(k^{\circ}, j^{\circ}\right)$ such that $\left(k^{\circ}, j^{\circ}\right)>(k, j)$ with respect to the lexicographic order. We show that the claim holds for the entries in the position $(k, j)$. Let $\left((k, j),\left(k_{1}, j_{1}\right), \ldots,\left(k_{p}, j_{p}\right)\right)$ and $\left((k, j),\left(k_{1}^{\prime}, j_{1}^{\prime}\right), \ldots,\left(k_{p^{\prime}}^{\prime}, j_{p^{\prime}}^{\prime}\right)\right)$ 
be the lacunary sequences that start from the position $(k, j)$ with respect to the Cauchon diagrams $C_{\tilde{A}}$ and $C_{\tilde{B}}$, respectively. Then by Proposition 5.1, $\tilde{a}_{k j}$ and $\tilde{b}_{k j}$ allow the following representations ${ }^{1}$

$$
\begin{gathered}
\tilde{a}_{k j}=\frac{\operatorname{det} A[k, \ldots, k+l \mid j, \ldots, j+l]}{\operatorname{det} A[k+1, \ldots, k+l \mid j+1, \ldots, j+l]}, \\
\tilde{b}_{k j}=\frac{\operatorname{det} B\left[k, \ldots, k+l^{\prime} \mid j, \ldots, j+l^{\prime}\right]}{\operatorname{det} B\left[k+1, \ldots, k+l^{\prime} \mid j+1, \ldots, j+l^{\prime}\right]},
\end{gathered}
$$

where $l$ and $l^{\prime}$ are defined as in Condition I.

Let $k+j$ be even; the proof of the case that $k+j$ is odd is parallel. Then the following three cases are possible:

Case 1: Suppose that $l=l^{\prime}$. Then by (5.14), (5.15), and Lemma 2.5, we have

$$
\tilde{a}_{k j} \leq \tilde{b}_{k j}
$$

Case 2: Suppose that $l<l^{\prime}$. By Lemma 4.2 and without loss of generality, we may assume that $d\left(j_{0}, j_{1}, \ldots, j_{l}\right)=0$. If $k=n-1$, then $l^{\prime}=1, l=0$. Hence, $\tilde{A}[n \mid 1, \ldots, j]=0$ or $\tilde{A}[1, \ldots, n-1 \mid$ $j+1, \ldots, m]=0$ which implies by Proposition 3.8 that $A[n \mid 1, \ldots, j]=0$ or $A[1, \ldots, n-1 \mid j+1, \ldots, m]=0$. In particular, $a_{n j}=0$ or $a_{n-1, j+1}=0$. Thus, $b_{n j}=0$ or $b_{n-1, j+1}=0$ since $n+j$ is odd and $A \leq^{*} B$ which implies that $B[n \mid 1, \ldots, j]=0$ or $B[1, \ldots, n-1 \mid j+1, \ldots, m]=0$. Therefore, $\tilde{B}[n \mid 1, \ldots, j]=0$ or $\tilde{B}[1, \ldots, n-1 \mid j+1, \ldots, m]=0$. Whence $l^{\prime}=0$ which is a contradiction. Let $h:=\min \left\{s: \tilde{a}_{k_{s}+1, j_{s}}=0\right\}$. The sequence $\left(\left(k_{h}+1, j_{h}\right),\left(k_{h+1}, j_{h+1}\right), \ldots,\left(k_{p}, j_{p}\right)\right)$ is a lacunary sequence since $d\left(j_{0}, j_{1}, \ldots, j_{\ell}\right)=0$. Because $\tilde{a}_{k_{h}+1, j_{h}}=0$ and $d\left(j_{0}, j_{1}, \ldots, j_{\ell}\right)=0$, we conclude by the induction hypothesis and Proposition 3.7 that

$$
\operatorname{det} A\left[k_{h}+1, k_{h}+2, \ldots, k_{h}+1+l-h \mid j_{h}, j_{h}+1, \ldots, j_{h}+l-h\right]=0 .
$$

Since $k_{h}=k+h$ and $j_{h}=j+h$, we obtain

$$
\operatorname{det} A[k+h+1, k+h+2, \ldots, k+1+l \mid j+h, j+h+1, \ldots, j+l]=0 .
$$

By Lemma 2.3, it follows that

$$
\operatorname{det} A[k+1, \ldots, k+l+1 \mid j, \ldots, j+l]=0,
$$

and consequently by Lemma 2.4,

$$
\operatorname{det} B[k+1, \ldots, k+l+1 \mid j, \ldots, j+l]=0
$$

since otherwise we would have $\operatorname{det} A[k+1, \ldots, k+l+1 \mid j, \ldots, j+l]>0$. By using Sylvester's Identity and again Lemma 2.3, we obtain

$$
\begin{aligned}
\tilde{b}_{k j}= & \frac{\operatorname{det} B\left[k, \ldots, k+l^{\prime} \mid j, \ldots, j+l^{\prime}\right]}{\operatorname{det} B\left[k+1, \ldots, k+l^{\prime} \mid j+1, \ldots, j+l^{\prime}\right]} \\
= & \frac{\operatorname{det} B\left[k, \ldots, k+l^{\prime}-1 \mid j, \ldots, j+l^{\prime}-1\right] \operatorname{det} B\left[k+1, \ldots, k+l^{\prime} \mid j+1, \ldots, j+l^{\prime}\right]}{\operatorname{det} B\left[k+1, \ldots, k+l^{\prime} \mid j+1, \ldots, j+l^{\prime}\right] \operatorname{det} B\left[k+1, \ldots, k+l^{\prime}-1 \mid j+1, \ldots, j+l^{\prime}-1\right]} \\
& -\frac{\operatorname{det} B\left[k, \ldots, k+l^{\prime}-1 \mid j+1, \ldots, j+l^{\prime}\right] \operatorname{det} B\left[k+1, \ldots, k+l^{\prime} \mid j, \ldots, j+l^{\prime}-1\right]}{\operatorname{det} B\left[k+1, \ldots, k+l^{\prime} \mid j+1, \ldots, j+l^{\prime}\right] \operatorname{det} B\left[k+1, \ldots, k+l^{\prime}-1 \mid j+1, \ldots, j+l^{\prime}-1\right]} \\
= & \frac{\operatorname{det} B\left[k, \ldots, k+l^{\prime}-1 \mid j, \ldots, j+l^{\prime}-1\right]}{\operatorname{det} B\left[k+1, \ldots, k+l^{\prime}-1 \mid j+1, \ldots, j+l^{\prime}-1\right]} .
\end{aligned}
$$

\footnotetext{
${ }^{1}$ If $l=0$ or $l^{\prime}=0$, we employ the convention that the respective denominator is 1 .
} 
If $l^{\prime}=l+1$, then $\tilde{b}_{k j}$ has order $l$. Otherwise, apply Sylvester's Identity repeatedly to obtain the required order.

Case 3: Suppose that $l^{\prime}<l$. Without loss of generality assume that $d\left(j_{0}^{\prime}, j_{1}^{\prime}, \ldots, j_{l^{\prime}}^{\prime}\right)=0$. Let $A_{1}:=$ $A[k+1 \ldots, k+l \mid j+1, \ldots, j+l]$ and $B_{1}:=B[k+1 \ldots, k+l \mid j+1, \ldots, j+l]$, then $A_{1}$ is $N s T N$ and $A_{1} \leq{ }^{*} B_{1}$. By Lemma 2.4, we obtain

$$
0<\operatorname{det} A_{1} \leq \operatorname{det} B_{1}
$$

We conclude that $B_{1}$ is nonsingular.

Let $h:=\max \left\{s \mid d\left(k_{0}^{\prime}, k_{1}^{\prime}, \ldots, k_{s}^{\prime}\right)=0\right\}$. Then define the sequence

$$
\left(\left(k_{h}^{\prime}+1, j_{h}^{\prime}\right),\left(k_{h+1}^{\prime}, j_{h+1}^{\prime}\right), \ldots,\left(k_{p^{\prime}}^{\prime}, j_{p^{\prime}}^{\prime}\right)\right)
$$

which is a lacunary sequence. By the induction hypothesis, $\operatorname{det} B\left[k_{h}^{\prime}+1, \ldots, k_{h}^{\prime}+l^{\prime} \mid j_{h}^{\prime}, \ldots, j_{h}^{\prime}+l^{\prime}-1\right]=0$. By Lemma 2.3, $\operatorname{det} B\left[k_{h}^{\prime}+1, \ldots, k_{h}^{\prime}+l^{\prime}+s \mid j_{h}^{\prime}, \ldots, j_{h}^{\prime}+l^{\prime}-1+s\right]=0, s=1,2, \ldots$

By using Sylvester's Identity if $l=l^{\prime}+1$, we obtain

$$
\begin{aligned}
\tilde{b}_{k j} & =\frac{\operatorname{det} B\left[k, k+1 \ldots, k+l^{\prime}+1 \mid j, j+1, \ldots, j+l^{\prime}+1\right]}{\operatorname{det} B\left[k+1, \ldots, k+l^{\prime}+1 \mid j+1, \ldots, j+l^{\prime}+1\right]} \\
& =\frac{\operatorname{det} B[k, k+1 \ldots, k+l \mid j, j+1, \ldots, j+l]}{\operatorname{det} B[k+1, \ldots, k+l \mid j+1, \ldots, j+l]} .
\end{aligned}
$$

If $l>l^{\prime}+1$, we apply Sylvester's Identity repeatedly to arrive at the required order.

TheOrem 5.3. Let $A, B, Z \in \mathbb{R}^{n, n}$ be such that $A \leq^{*} Z \leq^{*} B$. Let $A$, $B$ be TN and satisfy the descending rank conditions, and let $A^{\#}, B^{\#}$ satisfy Condition I. Then $Z$ is TN and satisfies the descending rank conditions.

Proof. Put $A_{1}:=A^{\#}, B_{1}:=B^{\#}, Z_{1}:=Z^{\#}$. Then $A_{1} \leq^{*} Z_{1} \leq^{*} B_{1}, A_{1}, B_{1}$ are $T N$, and by assumption, Condition I holds for both $A_{1}$ and $B_{1}$. Then by Theorem $3.3, \tilde{A}_{1}=\left(\tilde{a}_{i j}\right)$ and $\tilde{B}_{1}=\left(\tilde{b}_{i j}\right)$ are nonnegative Cauchon matrices and satisfy conditions (i)-(ii) in Theorem 3.14. By Theorems 3.3 and 3.15 it suffices to show that $\tilde{Z}_{1}$ is a nonnegative Cauchon matrix and satisfies conditions (i)-(ii) in Theorem 3.14.

By Theorem 5.2, $\tilde{a}_{i j}$ and $\tilde{b}_{i j}$ can be represented as ratios of contiguous minors of the same order, i.e.,

$$
\begin{aligned}
\tilde{a}_{i j} & =\frac{\operatorname{det} A_{1}[i, i+1 \ldots, i+\ell \mid j, j+1, \ldots, j+\ell]}{\operatorname{det} A_{1}[i+1, \ldots, i+\ell \mid j+1, \ldots, j+\ell]}, \\
\tilde{b}_{i j} & =\frac{\operatorname{det} B_{1}[i, i+1, \ldots, i+\ell \mid j, j+1, \ldots, j+\ell]}{\operatorname{det} B_{1}[i+1, \ldots, i+\ell \mid j+1, \ldots, j+\ell]}
\end{aligned}
$$

for some $\ell$. By Lemma 2.5,

$$
\tilde{A}_{1} \leq^{*} Z^{\prime} \leq^{*} \tilde{B}_{1}
$$

where $Z^{\prime}=\left(z_{i j}^{\prime}\right)$ with

$$
z_{i j}^{\prime}:=\frac{\operatorname{det} Z_{1}[i, i+1, \ldots, i+\ell \mid j, j+1, \ldots, j+\ell]}{\operatorname{det} Z_{1}[i+1, \ldots, i+\ell \mid j+1, \ldots, j+\ell]} .
$$

From (5.16) it follows that $Z^{\prime} \geq 0$. If $z_{i i}^{\prime}=0$, then by (5.16), $\tilde{a}_{i i}=0$. Since $A$ satisfies the descending rank conditions, we can apply Theorem 3.14 to conclude that $\tilde{a}_{s i}=\tilde{a}_{i t}=0, s, t=1, \ldots, i$. Again by (5.16), 
we conclude that $\tilde{b}_{i-1, i}=\tilde{b}_{i, i-1}=0$ and since $B$ satisfies the descending rank conditions, we obtain that $\tilde{b}_{i t}=\tilde{b}_{s i}=0, s, t=1, \ldots, i-1$. Hence, $z_{i t}^{\prime}=z_{s i}^{\prime}=0, s, t=1, \ldots, i$. We proceed in the same way if $z_{i j}^{\prime}=0$, $i<j$ or $i>j$, to obtain:

(i) If $z_{i j}^{\prime}=0$ for some $i \geq j$, then $z_{i t}^{\prime}=0$ for all $t<j$.

(ii) If $z_{i j}^{\prime}=0$ for some $i \leq j$, then $z_{t j}^{\prime}=0$ for all $t<i$.

Therefore, $Z^{\prime}$ is a Cauchon matrix. If we are able to show that $Z^{\prime}=\tilde{Z}_{1}$, then by Theorems 3.3 and 3.15 , we are done.

Claim: $Z^{\prime}=\tilde{Z}_{1}$. We proceed by decreasing induction with respect to the lexicographic order on the pairs $(i, j), i, j=1, \ldots, n$. By definition, $z_{n j}^{\prime}=z_{n j}=\tilde{z}_{n j}$ for all $j=1, \ldots, n$. Suppose that we have shown the claim for each pair $\left(i^{\circ}, j^{\circ}\right)$ such that $i^{\circ}=i+1, \ldots, n, j^{\circ}=1, \ldots, n$ and $i^{\circ}=i, j^{\circ}=j+1, \ldots, n$. Without loss of generality we may assume that $i+j$ is even. Let $\left((i, j),\left(i_{1}^{\prime \prime}, j_{1}^{\prime \prime}\right), \ldots,\left(i_{p_{1}}^{\prime \prime}, j_{p_{1}}^{\prime \prime}\right)\right)$ be a lacunary sequence with respect to $C_{Z^{\prime}}$ such that $\ell^{\prime \prime}$ is the minimum order and $d\left(i, i_{1}^{\prime \prime}, \ldots, i_{\ell^{\prime \prime}}^{\prime \prime}\right)=0$ or $d\left(j, j_{1}^{\prime \prime}, \ldots, j_{\ell^{\prime \prime}}^{\prime \prime}\right)=0$. Without loss of generality, assume that $d\left(j, j_{1}^{\prime \prime}, \ldots, j_{\ell^{\prime \prime}}^{\prime \prime}\right)=0$ and $i \geq j$. By (5.9) we have the following representation

$$
\tilde{z}_{i j}=\frac{\operatorname{det} Z_{1}\left[i, i_{1}^{\prime \prime}, \ldots, i_{\ell^{\prime \prime}}^{\prime \prime} \mid j, j_{1}^{\prime \prime}, \ldots, j_{\ell^{\prime \prime}}^{\prime \prime}\right]}{\operatorname{det} Z_{1}\left[i_{1}^{\prime \prime}, \ldots, i_{\ell^{\prime \prime}}^{\prime \prime} \mid j_{1}^{\prime \prime}, \ldots, j_{\ell^{\prime \prime}}^{\prime \prime}\right]}
$$

By Proposition 3.10, $\operatorname{rank}\left(Z_{1}\left[i_{1}^{\prime \prime}, i_{1}^{\prime \prime}+1, \ldots, n \mid j_{1}^{\prime \prime}, \ldots, j_{\ell^{\prime \prime}}^{\prime \prime}\right]\right)=\ell^{\prime \prime}$ since the lacunary sequence $\left(\left(i_{1}^{\prime \prime}, j_{1}^{\prime \prime}\right), \ldots\right.$, $\left.\left(i_{\ell^{\prime \prime}}^{\prime \prime}, j_{\ell^{\prime \prime}}^{\prime \prime}\right)\right)$ coincides with the one that is constructed by Procedure 3.9 applied to the columns $j_{1}^{\prime \prime}, \ldots, j_{\ell^{\prime \prime}}^{\prime \prime}$ of $Z^{\prime}$. Hence,

$$
Z_{1}\left[i+1, i+2, \ldots, i+\ell^{\prime \prime} \mid j+1, j+2 \ldots, j+\ell^{\prime \prime}\right]=C Z_{1}\left[i_{1}^{\prime \prime}, i_{2}^{\prime \prime}, \ldots, i_{\ell^{\prime \prime}}^{\prime \prime} \mid j_{1}^{\prime \prime}, j_{2}^{\prime \prime}, \ldots, j_{\ell^{\prime \prime}}^{\prime \prime}\right],
$$

for some $C \in \mathbb{R}^{\ell^{\prime \prime}, \ell^{\prime \prime}}$. We distinguish the following three cases:

Case 1: $\ell=\ell^{\prime \prime}$. We get from Lemma 2.4

$$
\begin{aligned}
0 & <\operatorname{det} A_{1}[i+1, i+2, \ldots, i+\ell \mid j+1, j+2 \ldots, j+\ell] \\
& \leq \operatorname{det} Z_{1}\left[i+1, i+2, \ldots, i+\ell^{\prime \prime} \mid j+1, j+2 \ldots, j+\ell^{\prime \prime}\right],
\end{aligned}
$$

and conclude that $\operatorname{det} C \neq 0$. Proceeding as in the proof of Proposition 5.1, we arrive at

$$
\tilde{z}_{i j}=\frac{\operatorname{det} Z_{1}[i, i+1 \ldots, i+\ell \mid j, j+1 \ldots, j+\ell]}{\operatorname{det} Z_{1}[i+1 \ldots, i+\ell \mid j+1 \ldots, j+\ell]}=z_{i j}^{\prime} .
$$

Case 2: $\ell^{\prime \prime}<\ell$. By Lemma 2.3,

$$
\operatorname{det} A_{1}\left[i+1, \ldots, i+\ell^{\prime \prime}+s \mid j+1, \ldots, j+\ell^{\prime \prime}+s\right]>0
$$

because $A_{1}\left[i+1, \ldots, i+\ell^{\prime \prime}+s \mid j+1, \ldots, j+\ell^{\prime \prime}+s\right]$ are leading principal submatrices in $A_{1}[i+1, \ldots, i+\ell \mid$ $j+1, \ldots, j+\ell]$ for all $s=0,1, \ldots, \ell-\ell^{\prime \prime}$. By Lemma 2.4,

$$
\operatorname{det} Z_{1}\left[i+1, \ldots, i+\ell^{\prime \prime}+s \mid j+1, \ldots, j+\ell^{\prime \prime}+s\right]>0, s=0,1, \ldots, \ell-\ell^{\prime \prime} .
$$

We proceed parallel to Case 1 to arrive at

$$
\begin{aligned}
\tilde{z}_{i j} & =\frac{\operatorname{det} Z_{1}\left[i, i_{1}^{\prime \prime}, \ldots, i_{\ell^{\prime \prime}}^{\prime \prime} \mid j, j_{1}^{\prime \prime}, \ldots, j_{\ell^{\prime \prime}}^{\prime \prime}\right]}{\operatorname{det} Z_{1}\left[i_{1}^{\prime \prime}, \ldots, i_{\ell^{\prime \prime}}^{\prime \prime} \mid j_{1}^{\prime \prime}, \ldots, j_{\ell^{\prime \prime}}^{\prime \prime}\right]} \\
& =\frac{\operatorname{det} Z_{1}\left[i, i+1, \ldots, i+\ell^{\prime \prime} \mid j, j+1, \ldots, j+\ell^{\prime \prime}\right]}{\operatorname{det} Z_{1}\left[i+1, \ldots, i+\ell^{\prime \prime} \mid j+1, \ldots, j+\ell^{\prime \prime}\right]}
\end{aligned}
$$


By the induction hypothesis, $Z_{1}[i+1, \ldots, n \mid j, j+1, \ldots, n]$ is $T N$. By arguing as in Case 3 in the proof of Theorem 5.2, we may conclude that $\operatorname{det} Z_{1}\left[i+1, \ldots, i+\ell^{\prime \prime}+1 \mid j, j+1, \ldots, j+\ell^{\prime \prime}\right]=0$. By Lemma 2.3, we have

$$
\operatorname{det} Z_{1}\left[i+1, \ldots, i+\ell^{\prime \prime}+1+s \mid j, j+1, \ldots, j+\ell^{\prime \prime}+s\right]=0, \quad s=1, \ldots, \ell-\ell^{\prime \prime}-1
$$

Application of Sylvester's Identity step by step to the representation of $\tilde{z}_{i j}$ that is given in (5.18), we obtain

$$
\begin{aligned}
\tilde{z}_{i j} & =\frac{\operatorname{det} Z_{1}\left[i, i+1, \ldots, i+\ell^{\prime \prime} \mid j, j+1, \ldots, j+\ell^{\prime \prime}\right]}{\operatorname{det} Z_{1}\left[i+1, \ldots, i+\ell^{\prime \prime} \mid j+1, \ldots, j+\ell^{\prime \prime}\right]} \\
& =\frac{\operatorname{det} Z_{1}\left[i, i+1, \ldots, i+\ell^{\prime \prime}+1 \mid j, j+1, \ldots, j+\ell^{\prime \prime}+1\right]}{\operatorname{det} Z_{1}\left[i+1, \ldots, i+\ell^{\prime \prime}+1 \mid j+1, \ldots, j+\ell^{\prime \prime}+1\right]} \\
& \vdots \\
& =\frac{\operatorname{det} Z_{1}[i, i+1, \ldots, i+\ell \mid j, j+1, \ldots, j+\ell]}{\operatorname{det} Z_{1}[i+1, \ldots, i+\ell \mid j+1, \ldots, j+\ell]} \\
& =z_{i j}^{\prime} .
\end{aligned}
$$

Case 3: $\ell<\ell^{\prime \prime}$. Define $W:=Z_{1}\left[i+1, i+2, \ldots, i+\ell^{\prime \prime} \mid j+1, j+2 \ldots, j+\ell^{\prime \prime}\right]$. If $\operatorname{det} W \neq 0$, then $\tilde{z}_{i j}$ can be written as in (5.18). Otherwise, by [15, Proposition 1.15] the rows $i+1, \ldots, i+\ell^{\prime \prime}$ of $Z_{1}$ are linearly dependent or the right shadow of $W$ in $Z_{1}[i+1, i+2, \ldots, n \mid 1,2 \ldots, m]$ has rank at most $\ell^{\prime \prime}-1$ since by the induction hypothesis the later submatrix is $T N$ and $d\left(j, j_{1}^{\prime \prime}, \ldots, j_{\ell^{\prime \prime}}^{\prime \prime}\right)=0$. If $i=j$, then define $\left(\alpha_{0}, \beta_{0}\right):=(i, j)$ and for $k=1, \ldots, \tau$, let

$$
\left(\alpha_{k}, \beta_{k}\right):=\min \left\{(\alpha, \beta) \mid \alpha=\alpha_{k-1}+1, \beta>\beta_{k-1}, z_{\alpha, \beta}^{\prime} \neq 0\right\}
$$

where the minimum is taken with respect to the lexicographic order. This sequence is a lacunary sequence or a part of a lacunary sequence of order $\tau$ since the entries of $Z^{\prime}$ satisfy the conditions (i) and (ii) above with possible gaps between columns and $\tau<\ell^{\prime \prime}$ which is a contradiction. Hence, if $i=j$, then det $W \neq 0$. If $i>j$, then $j<i-1$ since $i+j$ is even. It is easy to see that the order of the sequence at the position $(i, j)$ is less than or equal to that of $(i, j+1)$. Hence, by the induction hypothesis, the rows $i+1, \ldots, i+\ell^{\prime \prime}$ cannot be linearly dependent and the right shadow of $W$ in $Z_{1}[i+1, i+2, \ldots, n \mid 1,2 \ldots, m]$ has not rank less than $\ell^{\prime \prime}$. Thus, $\operatorname{det} W \neq 0$ and we conclude that $\operatorname{det} C \neq 0$. Therefore, $\tilde{z}_{i j}$ can be written as in (5.18). Proceeding as in the proof of Theorem 5.2, Case 2 and by Lemma 2.3, we arrive at

$$
\operatorname{det} Z_{1}[i+1, \ldots, i+\ell+1+s \mid j, \ldots, j+\ell+s]=0, \quad s=0,1, \ldots, \ell^{\prime \prime}-\ell-1
$$

Now use Sylvester's Identity to decrease step by step the order of the representation similarly as in (5.19) to obtain $\tilde{z}_{i j}=z_{i j}^{\prime}$. This completes the proof.

TheOREM 5.4. Let $A, B, Z \in \mathbb{R}^{n, m}$ be such that $A \leq^{*} Z \leq^{*} B$. If $A, B$ are $T N$, belong to the same TN cell, and both satisfy Condition $I$, then $Z$ is TN, satisfies Condition $I$, and belongs to the same $T N$ cell that includes $A$ and $B$.

The proof of this theorem is parallel to the proof of the Theorem 5.3 and therefore omitted.

The follwing example illustrates the difference between Theorem 5.3 and Theorem 5.4. 
EXAMPLE 5.5. Let

$$
A=\left[\begin{array}{lll}
1 & 1 & 1 \\
2 & 3 & 3 \\
2 & 3 & 3
\end{array}\right], \quad B=\left[\begin{array}{lll}
1 & 1 & 1 \\
2 & 3 & 3 \\
2 & 3 & 7
\end{array}\right] \quad \text { and } \quad Z=\left[\begin{array}{lll}
1 & 1 & 1 \\
2 & 3 & 3 \\
2 & 3 & 4
\end{array}\right]
$$

Then we have

$$
A \leq^{*} Z \leq^{*} B
$$

and obtain

$$
\tilde{A}=\left[\begin{array}{ccc}
\frac{1}{3} & 0 & 1 \\
0 & 0 & 3 \\
2 & 3 & 3
\end{array}\right] \quad \text { and } \quad \tilde{B}=\left[\begin{array}{ccc}
\frac{1}{3} & 0 & 1 \\
0 & \frac{12}{17} & 3 \\
2 & 3 & 7
\end{array}\right]
$$

$A, B$ are $T N$ but belong to two different TN cells and satisfy the descending rank conditions. $A^{\#}, B^{\#}$ fulfill Condition I. $Z$ is TN.

In [2], two relaxations of the nonsingularity assumption are presented. The following example shows that Theorem 5.3 covers a different situation.

EXAMPLE 5.6. Let

$$
A=\left[\begin{array}{ccc}
1 & 2 & 1 \\
5 & 10 & 5 \\
1 & 2 & 1
\end{array}\right] \quad \text { and } B=\left[\begin{array}{ccc}
2 & 2 & 1 \\
5 & 10 & 5 \\
1 & 2 & 13
\end{array}\right]
$$

Then we have

$$
A \leq^{*} B
$$

and obtain

$$
\tilde{A}=\left[\begin{array}{lll}
0 & 0 & 1 \\
0 & 0 & 5 \\
1 & 2 & 1
\end{array}\right] \text { and } \quad \tilde{B}=\left[\begin{array}{ccc}
1 & 0 & 1 \\
0 & \frac{120}{13} & 5 \\
1 & 2 & 13
\end{array}\right]
$$

$A$ and $B$ are $T N$, both $A\left(=A^{\#}\right)$ and $B\left(=B^{\#}\right)$ satisfy Condition I as well as the descending rank conditions. Hence, all matrices in $[A, B]$ are $T N$. Neither [2, Theorem 3.6] nor [2, Corollary 3.7] can be used to draw this conclusion since $A$ is singular and

$$
\operatorname{det} A[1,2]=\operatorname{det} A[2,3]=0 .
$$

Unfortunately, Condition I alone is not strong enough to guarantee the interval property as the following example documents.

EXAMPLE 5.7. Let

$$
A=\left[\begin{array}{llll}
3 & 2 & 2 & 2 \\
6 & 5 & 5 & 5 \\
3 & 3 & 3 & 3
\end{array}\right], \quad Z=\left[\begin{array}{llll}
4 & 2 & 2 & 1 \\
6 & 5 & 5 & 5 \\
3 & 3 & 3 & 3
\end{array}\right] \quad \text { and } \quad B=\left[\begin{array}{llll}
5 & 2 & 2 & 1 \\
5 & 5 & 5 & 5 \\
3 & 3 & 3 & 3
\end{array}\right]
$$

$A$ and $B$ are $T N$, satisfy Condition I, and $A \leq^{*} Z \leq^{*} B$. But $Z$ is not $T N$ since $\operatorname{det} Z[1,2,3 \mid 1,2,4]=$ $-3<0$. 
Acknowledgment. The work on this paper was finalized during M. Adm's stay in the period May July 2019 at the University of Konstanz which was funded by the Arab-German Young Academy of Sciences and Humanities (AGYA). The work of M. Adm leading to this publication was started during his work at the University of Konstanz in 2018, where he was supported by the German Academic Exchange Service (DAAD) with funds from the German Federal Ministry of Education and Research (BMBF) and the People Programme (Marie Curie Actions) of the European Union's Seventh Frame-work Programme (FP7/20072013) under REA grant agreement no. 605728 (P.R.I.M.E. - Postdoctoral Researchers International Mobility Experience) and continued during his work at Palestine Polytechnic University.

\section{REFERENCES}

[1] M. Adm. Perturbation and Intervals of Totally Nonnegative Matrices and Related Properties of Sign Regular Matrices. Dissertation, University of Konstanz, Konstanz, Germany, 2016.

[2] M. Adm and J. Garloff. Intervals of totally nonnegative matrices. Linear Algebra Appl., 439:3796-3806, 2013.

[3] M. Adm and J. Garloff. Invariance of total nonnegativity of a tridiagonal matrix under element-wise perturbation. Oper. Matrices, 8:129-137, 2014.

[4] M. Adm and J. Garloff. Improved tests and characterizations of totally nonnegative matrices. Electron. J. Linear Algebra, $27: 588-610,2014$.

[5] M. Adm and J. Garloff. Intervals of special sign regular matrices. Linear Multilinear Algebra, 64:1424-1444, 2016.

[6] M. Adm and J. Garloff. Invariance of total positivity of a matrix under entry-wise perturbation and completion problems. In: C.M. da Fonseca, D. Van Huynh, S. Kirkland, and V.K. Tuan (editors), A Panorama of Mathematics: Pure and Applied, Contemp. Math., Amer. Math. Soc., Providence, RI, 658:115-126, 2016.

[7] M. Adm and J. Garloff. Invariance of total nonnegativity of a matrix under entry-wise perturbation and subdirect sum of totally nonnegative matrices. Linear Algebra Appl., 541:222-233, 2017.

[8] M. Adm, K. Muhtaseb, A. Ghani, S. Fallat, and J. Garloff. Further applications of the Cauchon algorithm to rank determination and bidiagonal factorization. Linear Algebra Appl., 545:240-255, 2018.

[9] S.M. Fallat and C.R. Johnson. Totally Nonnegative Matrices. Princeton University Press, Princeton, 2011.

[10] S.M. Fallat, C.R. Johnson, and R.L. Smith. The general totally positive matrix completion problem with few unspecified entries. Electron. J. Linear Algebra, 7:1-20, 2000.

[11] J. Garloff, M. Adm, and J. Titi. A survey of classes of matrices possessing the interval property and related properties. Reliab. Comput., 22:1-10, 2016.

[12] K.R. Goodearl, S. Launois, and T.H. Lenagan. Totally nonnegative cells and matrix Poisson varieties. Adv. Math., 226:779-826, 2011.

[13] M. Hladík. Tolerances, robustness and parametrization of matrix properties related to optimization problems. Optimization 68:667-690, 2019.

[14] S. Launois and T.H. Lenagan. Efficient recognition of totally nonnegative matrix cells. Found. Comput. Math., 14:371-387, 2014.

[15] A. Pinkus. Totally Positive Matrices. Cambridge University Press, Cambridge, 2010. 